Collision course
Physicists get excited
over possible sighting
of Higgs boson
$p 118$

\title{
Tests fail to support claims for origin of AlDS in polio vaccine
}

David Dickson, London

The hypothesis that AIDS has its origins in a contaminated polio vaccine used in Africa in the late 1950s suffered a significant knock this week. Tests on samples of the vaccine, in storage for over 40 years, have shown no trace of HIV or its primate antecedent SIV.

The tests were conducted at three different laboratories in the United States, Germany and France (see below). The work was instigated by the Wistar Institute in Philadelphia, which has been at the centre of the vaccine controversy since it was first raised almost a decade ago.

According to the eagerly awaited results, which were revealed at a two-day meeting on the origins of AIDS held at the Royal Society in London, none of the laboratories found any trace of HIV or SIV in their samples.

In addition, studies of mitochondrial DNA from the samples failed to provide any evidence to support allegations that the polio vaccine had been prepared using chimpanzee tissue - known to be the source of the HIV virus. In each case, the material used to produce the polio virus for the vaccine was shown to be of monkey origin.

The Wistar Institute claims that the results not only vindicate its own role in the issue, but will also soothe public concern

\section{Weighing the evidence}

The tests on samples of polio vaccine produced by the Wistar Institute in Philadelphia in the 1950s (see above) were carried out at three independent laboratories, which also tested additional sets of samples and controls prepared by the Centers for Disease Control and Prevention in Atlanta, Georgia. A research group at Roche Molecular Systems in Pleasanton, California, headed by Shirley Kwok, tested the samples for the presence of SIV/HIV, and a second, headed by Svante Pääbo at the Max Planck Institute for Evolutionary Anthropology in Leipzig, Germany, tested for the presence and species origin of mitochondrial DNA. A third laboratory headed by Simon Wain-Hobson of the Pasteur Institute in Paris, France - one of the organizers of this week's meeting at the Royal Society in London - performed both sets of tests.

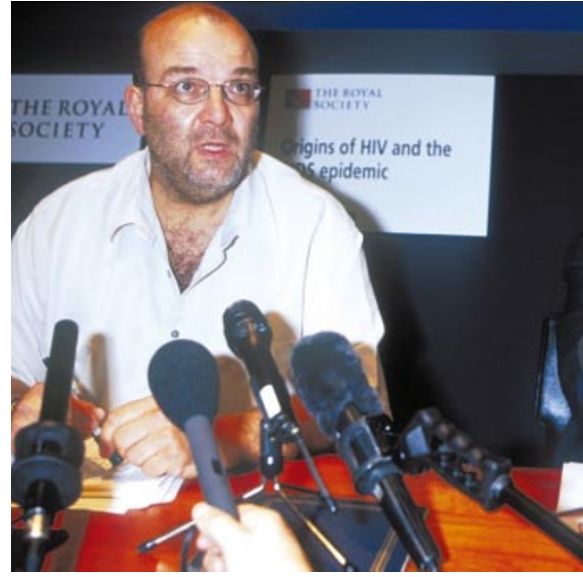

over the safety of vaccines, which had been raised by the allegations.

"We trust that these results will put to rest any remaining concerns of a link between a Wistar-produced oral polio vaccine and AIDS," Clayton Buck, its acting director, said in a statement. "The findings should also serve to restore public confidence in the production and administration of vaccines."

But writer Edward Hooper, the principal proponent of the 'contaminated vaccine' theory, told the Royal Society meeting that the findings made little impact on his commitment to the theory, expounded last year in his widely publicized book The River.

"I applaud Wistar's decision to release batches of vaccine for testing," Hooper said. "But different batches of vaccine were often produced in different laboratories." Vaccine samples released did not include any from batches prepared for use in Africa, he said.

Although this week's meeting gave detailed attention to all aspects of the origins of AIDS — including the opposing 'cuthunter' thesis that the virus may have passed from chimpanzees to humans through hunting and perhaps eating contaminated animals — it was prompted largely by the public controversy surrounding the contaminated vaccine theory (see Nature 404, 9; 2000).

Hooper told the meeting that in his own visits to Africa over the past eight weeks, he had come up with two "smoking guns". Both were individuals who claimed they knew that chimpanzee kidneys were being sent to

^ ๔ 2000 Macmillan Magazines Ltd
Best of enemies: Hooper (left) claims that chimpanzee kidneys may have been used secretly to make an experimental polio vaccine in the 1950s. Plotkin (right) argues that there is "not a shred of evidence" to support such an allegation. 